\title{
The Effects of Chromatic Aberration on the Performance of GalnP/GaAs/Ge Concentrator Solar Cells from Fresnel Optics
}

\author{
H. Cotal, and R. Sherif \\ Spectrolab, Inc. (A Boeing Company), 12500 Gladstone Ave, Sylmar, CA 91342 USA
}

\begin{abstract}
Results are reported for the effects of chromatic aberration on the electrical performance of triple junction (3J), GalnP/GaAs/Ge concentrator solar cells for indoor measurements. A Fresnel lens was moved away from the solar cell such that the concentration level increased, and then decreased while the lens-to-cell distance continued to increase. From such movement, the FF exhibited a series of minima and maxima in the region where light is apparently focused as determined by the naked eye. These effects are due to chromatic aberrations that affect the FF, and to some extent, the subcell short circuit currents in the $3 \mathrm{~J}$ stack. Aberrations can drive the $3 \mathrm{~J}$ device in a top cellor middle cell-limited mode, and make the top cell FF become more predominant than the middle cell FF, or vice versa with the $3 \mathrm{~J} \mathrm{FF}$ influenced more by either subcell FF. It is shown that the multijunction cell power output is also affected, and the gain in power depends on the size of the solar cell relative to the size of the beam waist, and the focal length of blue light relative to that of red light.
\end{abstract}

\section{INTRODUCTION}

The continuing development of triple junction (3J) GalnP/GaAs/Ge concentrator solar cells is making this technology closer to maturity and commercialization. It is recognized as the solar cell technology that produces the most $\mathrm{W} / \mathrm{cm}^{2}$ at 500 suns than any other while progress in boosting the efficiency keep rising. Already, there has been another record efficiency of $37.3 \%$ at 175 suns that was recently reported, as there have been other reports, that are continuing to stream in, from field tests on their reliability [13]. Successful demonstration of $3 \mathrm{~J}$ concentrators operating at 1000 suns under steady state illumination has been published [4,5] as well. However, one area that needs further studying to increase solar cell performance is in the system optics of the module, that is, Fresnel optics. Some studies focusing on improving Fresnel lenses to increase multijunction cell performance have been documented for dual junction GalnAs/GaAs concentrator cells [6].

\section{BACKGROUND}

Spectrolab has long been aware of optical dispersion in Fresnel optics and its effect on the electrical performance of multijunction concentrator solar cells. Light that traverses through a dispersive medium is subject to refraction in that each color of light travels through a specific optical path length so that when the rays exit the material they converge at different points in space. This is mainly due to the change in index of refraction of the material with wavelength which gives rise to chromatic aberration. Chromatic aberration can be manifested as axial and/or lateral aberration.
Axial chromatic aberration takes place when each color of light is focused at a different focal length. Equation (1) shows the change in focal length as a function index of refraction, namely

$$
\frac{d f}{f}=-\frac{d n(\lambda)}{n(\lambda)-1}
$$

This equation stems from Snell's law in differential form where $f$ is the focal length of the lens, $n$ is the index of refraction and $\lambda$ the wavelength. Figure 1 shows an exaggerated depiction of the case in point. When sunlight or simulated sunlight is focused on a test plane, the sharp white image formed on the test plane generally signifies that the image is in focus. In reality, however, each color of light is focused at different focal lengths. Typically for glass and many commercially available plastics, $n$ increases with a decrease in $\lambda$, and implies that $f$ becomes large, as $n$ increases.

In lateral aberration, the $\lambda$ dependence of the axial focal length causes a $\lambda$ dependence of the magnification in the transverse direction of the test plane. As a result, this causes the off-axis formation of different colors of light from the lens optical axis. As shown below, lens distortion can also affect the off-axis formation of focused light.

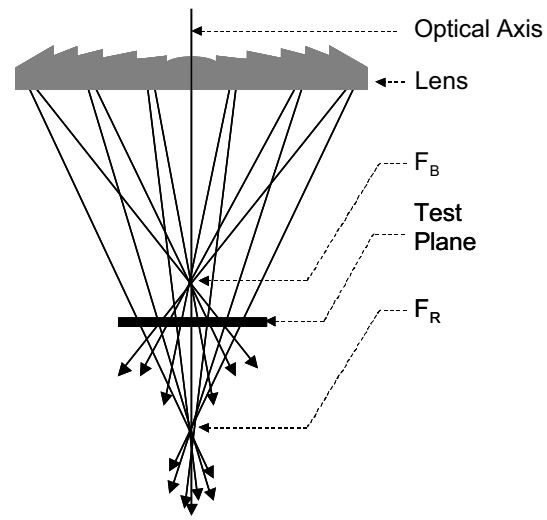

Figure 1: Axial chromatic aberration. Only 2 sets of color rays are shown for clarity. In reality each color of light has its own point of convergence. $F_{B}$ is the focal length of blue light and $F_{R}$ is the focal length for red light.

\section{Lens Distortion}

Another factor that can affect lateral chromatic aberration is distortion of a Fresnel lens. The amount of distortion depends on its thickness and material. Preferably thin lenses are desirable to reduce absorption losses that can affect the performance of multijunction solar cells but they can, however, compromise the rigidity of the lens. Rigidity depends on size and quality. The lens used in this 
study is somewhat distorted. The side with the facets is slightly concave in shape from extensive use. This is in addition to the thinner portion of the center of the lens due to the design and geometry of the facets. A simple exercise can be performed to estimate the degree of distortion from a concave-shaped lens.

Figure 2 depicts a diagram showing the amount of flex of an Edmund Scientific Fresnel lens that was used to obtain the IV concentration results.

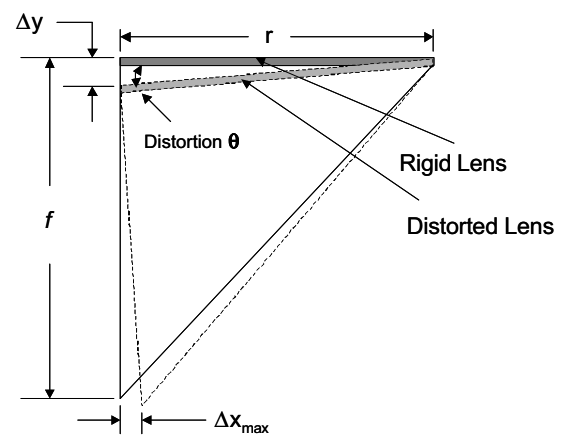

Figure 2: Half section of a Fresnel lens showing distortion. For a slightly distorted lens, $\Delta \mathrm{x}$ is the increase in the beam waist.

Here $\Delta y$ is the distance of distortion from the center of lens, $r$ is the lens radius, $f$ the focal length of the lens, $\theta$ the distortion angle and $\Delta x_{\max }$ is the maximum spread of the beam waist in the transverse direction. To a first order approximation, the spread in beam waist is estimated to be

$$
\Delta x_{\max }=r(1-\cos \theta)+f \sin \theta
$$

where $\theta=\tan ^{-1}(\Delta y / r)$.

There are several materials available for Fresnel lenses such as the commonly known acrylic, polycarbonate and rigid vinyl that usually transmit light from 300 to 2700 $\mathrm{nm}$. Another type of Fresnel lens is the color-mixing lens that has been proposed to evaluate the electrical performance of multijunction solar cells [7]. It is claimed this lens eliminates lateral chromatic aberration but no reports have demonstrated this. The size of multijunction concentrator solar cells can be susceptible to the aberrations discussed above. The size of the solar cell relative to the size of focal point is important, as is the focal length of blue light relative to that of red light

\section{CELL DETAILS AND MEASUREMENT DESCRIPTION}

Triple junction $n^{+} p$ GalnP ${ }_{2} / G a A s / G e$ epitaxial layers were grown on $\mathrm{Ge}$ wafers in a production MOVPE reactor at Spectrolab. Some details of the solar cell structure have been described elsewhere [8]. Indoor light IV measurements were performed at Spectrolab using an X-25 solar simulator with the filtered spectrum adjusted for AM1.5D. The spectrum was calibrated against $3 \mathrm{~J}$ iso-type structures measured at NREL with the short circuit current $\left(I_{\mathrm{sc}}\right)$ from each iso-type top and middle cells serving as the parameter to adjust the simulator intensity

\section{RESULTS AND DISCUSSION}

The $n^{+} p$ junction of each subcell in the multijunction stack has its own spectral response. For multijunction structures grown in a series-connected configuration, it is well known that the current produced from each junction must be balanced to their design criterion for the device to operate efficiently. This means that each GainP, GaAs and Ge subcell must absorb the appropriate amount of light to generate the short circuit current $\left(\mathrm{I}_{\mathrm{sc}}\right)$ needed to match the $\mathrm{I}_{\mathrm{sc}}$ of their counterpart subcells. Anything other than this will degrade device performance. Such degradation can be induced from a Fresnel lens.

\section{Fresnel Lens Transmission}

Light absorption and chromatic aberration from a Fresnel lens can affect the performance of $3 \mathrm{~J}$ solar cells by starving a subcell from the required amount of sunlight it needs to operate. Figure 3 shows spectral response from nominal concentrator solar cells together with the transmission characteristics of an inexpensive, Edmond Scientific, $0.25 \mathrm{~cm}$ thick, acrylic Fresnel lens that is used for indoor testing with the X25 solar simulator. Although the top and middle subcells are enjoying absorption of light from the highly transmissive portion of the visible and near infrared (IR) from the lens, light absorption farther in the IR shows 2 absorption bands at 1175 and $1395 \mathrm{~nm}$ that could reduce the $\mathrm{Ge}$ subell $\mathrm{I}_{\mathrm{sc}}$. However, integrating its spectral response with the AM1.5 spectrum, the $\mathrm{I}_{\mathrm{sc}}$ of the Ge subcell is not dramatically affected. Care must be exercised in selecting thick lenses. Aside from absorption, and noting from time-to-time, the electrical performance varies with the distance between the cell and the lens. This is due to chromatic aberration.

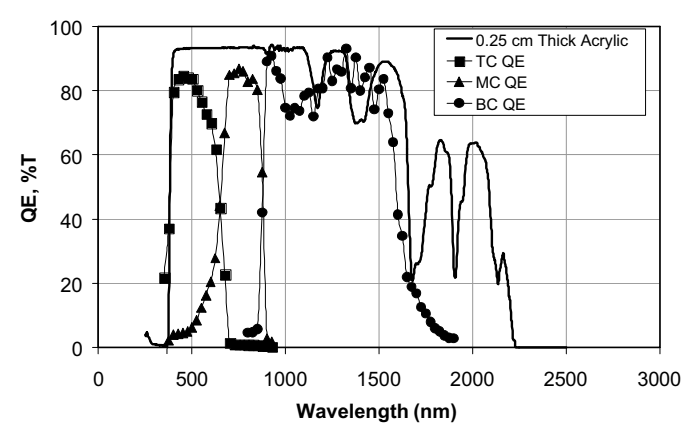

Figure 3: Nominal GalnP, GaAs and $\mathrm{Ge} S R$ and transmission of a $0.25 \mathrm{~cm}$ thick Fresnel lens with aperture area of $680 \mathrm{~cm}^{2}$.

\section{Chromatic Aberration}

Figure 4 illustrates the variation of the solar cell fill factor (FF) with an increase in concentration level. When a $0.25 \mathrm{~cm}$ thick Fresnel lens is used in conjunction with Spectrolab's X25 solar simulator, the intensity is such that concentration levels as high as 300 suns for a $0.306 \mathrm{~cm}^{2}$ and approximately 210 suns for $1 \mathrm{~cm}^{2}$ cell active areas can be achieved. Analysis of the FF at higher concentrations could not be achieved due to constraints in the test instrumentation. 


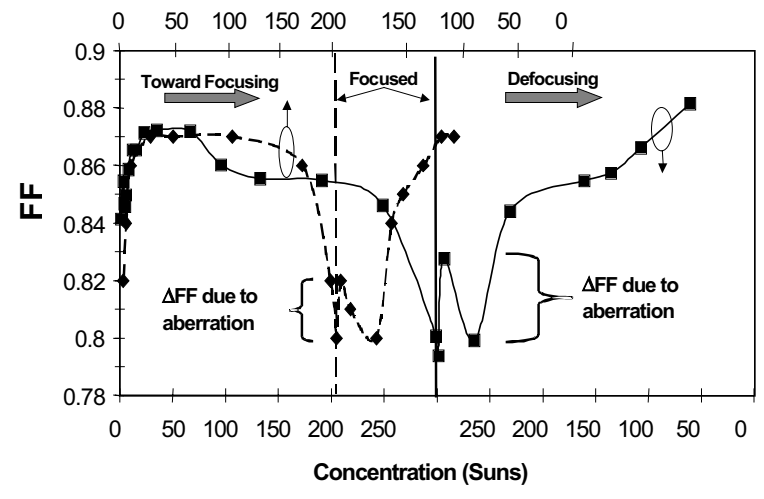

Figure 4: The effects of concentration on the FF for a $1 \mathrm{~cm}^{2}$ cell (dashed line) and a $0.306 \mathrm{~cm}^{2}$ cell (solid line.) The vertical dashed and solid lines indicate that light is entirely focused at these points for the dashed and solid curves, respectively. The lower axis represents the solid curve and the upper axis the dashed curve.

Movement of the lens away from the solar cell allows light to gradually come in focus on the cell until it is entirely focused as perceived by the naked eye, and the FF is apparently at a minimum due mostly in part to series resistance effects. For the cell represented by the dashed line in the figure, this minimum occurs at 209 suns. For the cell with the solid line, the minimum occurs at 298 suns. Entirely focused light is described as "focused" in the region of the figure between the dash and solid vertical lines. Further movement of the lens away from the cell as denoted by the "defocusing" label, the data exhibits a maximum in FF and then decreases again followed by a gradual increase toward low concentrations. The difference between the minimum FF when light is focused and the first maximum is denoted as $\Delta \mathrm{FF}$ for both plots.

Figure 5 shows the absolute efficiency for nominal $3 \mathrm{~J}$ concentrator solar cells when the behavior of the FF shown in Fig. 4 is present. The same "W"-like minima that occur in the FF also occur in the efficiency plots. $\Delta F F$, and consequently $\Delta \mathrm{Eff}$, has been seen in multiple tests when the Edmond Scientific lens is used. It is suggested that $\Delta \mathrm{FF}$ (and $\Delta \mathrm{Eff}$ ) is due to the combined effects of axial and lateral chromatic aberrations.

\section{Effect of Chromatic Aberration and Distortion on 3J FF}

The variation in FF due to chromatic aberration can be described with the aid of Figs. 6 and 1 . When light is almost focused, shorter wavelength light (blue bands) impinges on the cell while longer wavelength light (red bands) strikes just outside the cell as shown in Fig. 6a. Here the excess of blue light drives the $3 \mathrm{~J}$ cell in a middle cell-limited mode with the 3J FF primarily influenced by the FF of the middle cell. For a slightly defocused beam, that is, the lens moved further away from the cell by a small amount, blue light partly falls on the cell while some of it misses the cell as shown in Fig. 6b. At the same time, the red light bands begin to converge on the cell due to the aberration effects depicted in Fig. 1. In this case, the lack of blue light drives the $3 \mathrm{~J}$ cell in a top cell-limited mode with the overall FF mostly dominated by that of the top cell. When lens distortion is taken into account, aberrations can be exasperated. For the present lens, $\Delta y=0.1 \mathrm{~cm}, r=13$ $\mathrm{cm}$ and $f=17.8 \mathrm{~cm}$, thus the additional spread in the beam waist is $\Delta x_{\max }=0.14 \mathrm{~cm}$. This adds to lateral aberration and affects the cell performance. Because of axial and lateral aberrations, along with lens distortion, these act in concert with the cell series resistance to give the "W"-like feature that is observed in the FF and efficiency.

The mismatch in the $\mathrm{I}_{\mathrm{sc}}$ between the top cell (TC) and the middle cell (MC) due to aberrations also affect the performance of $3 \mathrm{~J}$ concentrators. This has been described in some detail for dual junction GalnAs/GaAs solar cells [6]. Aberration effects on the open circuit voltage play a minor role and do not influence the $3 \mathrm{~J}$ cell performance.

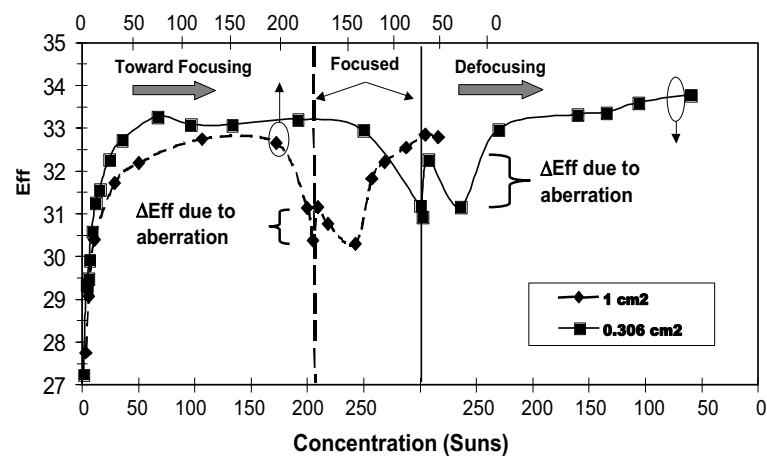

Figure 5: The effects of concentration on the efficiency as a result of changes in the $\mathrm{FF}$ for a $0.25 \mathrm{~cm}$ thick Fresnel lens with aperture area of $680 \mathrm{~cm}^{2}$. The terms have the same meaning as the caption in Fig. 4.

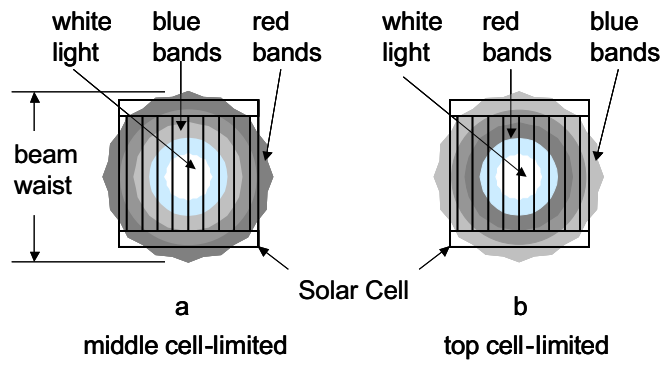

Figure 6: (a) Beam focused: blue light impinging on the cell, and some red light hitting outside of cell. (b) Beam slightly defocused: red light impinging on the cell while some blue light hitting outside of cell.

Worthy of mention are the low concentration 3J FF endpoints just when the lens begins to move and just before the lens stops to move. When the lens begins to move for the $0.306 \mathrm{~cm}^{2}$ cell, the FF values are near $87 \%$. Just before the lens stops, the FF has increased to approximately $88 \%$. This is due to influence of the subcell FFs on the 3J FF with the higher FF endpoint due to top cell-limited conditions from the deficient blue light. To help explain and understand the behavior of the FF as a function of concentration for the 3J concentrator cell, Fig. 7 shows the change in the $\mathrm{FF}$ for an electrically active, $1 \mathrm{~cm}^{2}$, GalnAs, 3J iso-type TC. Three measurements were performed on this cell where the lens was carefully moved away from the solar cell to focus and defocus the simulator light. Each plot shows an erratic trend in FF from measurement to measurement. Figure 8 illustrates the change in FF when the lens increases in distance from an electrically active, $1 \mathrm{~cm}^{2}$, GaAs, $3 \mathrm{~J}$ iso-type MC. Three 
measurements were also carried out carefully as shown by the 3 plots in the graph. An erratic pattern in FF is also seen for this cell. Electrically active, $1 \mathrm{~cm}^{2} \mathrm{Ge} 3 \mathrm{~J}$ iso-type bottom cells were measured as well but no discernable change in the FF was observed. Each iso-type TC and MC is sensitive to aberrations. Therefore, with the results presented thus far in this study, it can be concluded that the 3J FF is also sensitive to aberrations.

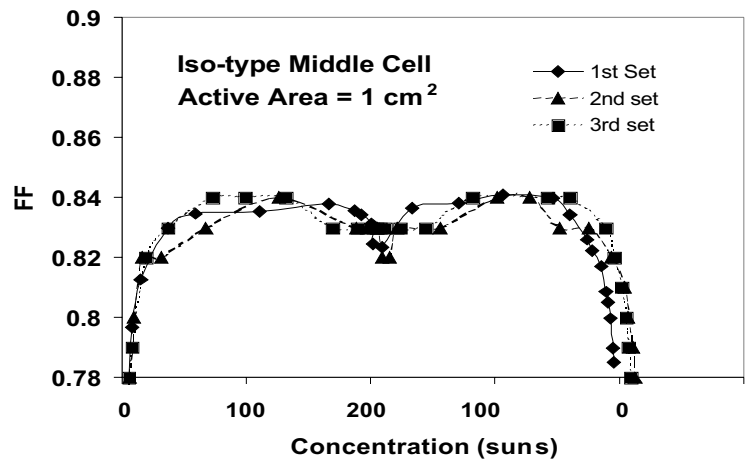

Figure 7: FF-dependence from multiple measurements for a GalnAs iso-type TC. No reproducibility is obtained as a result of accurate movement of the lens for each test.

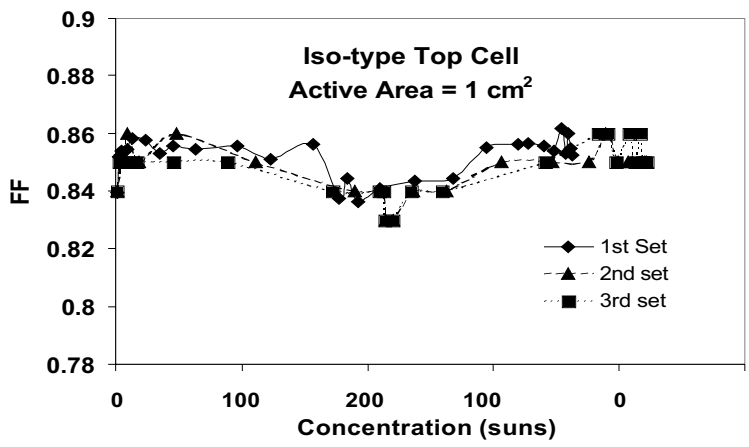

Figure 8: FF-dependence from multiple measurements for a GaAs iso-type MC. No reproducibility is obtained as a result of accurate movement of the lens for each test.

Although no data on the analysis of the change of the FF with increased concentration level for the smaller 0.306 $\mathrm{cm}^{2}$ iso-type TC and MC cells were presented, it is important to note that the change in FF was more pronounced for this cell than the $1 \mathrm{~cm}^{2}$ cell. This is due to the size of the beam waist when light is focused on the $0.306 \mathrm{~cm}^{2}$ cell. Since the beam waist for focused light is about $0.7 \mathrm{~cm}$ in diameter, the $0.306 \mathrm{~cm}^{2}$ cell is more sensitive to changes in the beam waist than the $1 \mathrm{~cm}^{2}$ cell, and therefore the FF changes more dramatically. In principle, for a lens with a relatively high degree of chromatic aberrations, it could be used in a slightly defocused mode to produce the highest performance from the solar cells. This method could be prone to errors if tracking errors and mounting errors are significant.. As an example, Table I shows the gain in maximum power as a function of FF for the case when the lens is focused and then defocused. For cells no. 1 and 2, there is an increase in power with a corresponding increase in $\mathrm{FF}$ as the concentration is increased with a $\Delta \mathrm{FF} \%=2 \%$ and $4.4 \%$, respectively. This is counterintuitive, as the FF should increase with decreasing concentration level when the lens is moved farther away from the cell. It is interesting to note that the gain in power for cells 1 and 2 of $6.5 \%$ and $2.6 \%$, respectively, does not correspond to the same increase in $\Delta F F$. This discrepancy is due to the increased mismatch of $I_{\text {sc }}$ between the TC and the MC subcells.

Table I: Maximum power and FF fluctuation as a result of chromatic aberration. Cell no. 1 represents the $1 \mathrm{~cm}^{2}$ cell whereas cell no. 2 is the $0.306 \mathrm{~cm}^{2}$ cell.

\begin{tabular}{ccccc}
\hline $\begin{array}{c}\text { Cell } \\
\text { No }\end{array}$ & Concentration & FF & $\begin{array}{c}\text { Power } \\
(\mathrm{W})\end{array}$ & $\begin{array}{c}\text { Mode } \\
\text { (F=focused, } \\
\text { D=defocused) }\end{array}$ \\
\hline \hline 1 & 200 & 0.807 & 5.22 & $\mathrm{~F}$ \\
1 & 209 & 0.823 & 5.56 & $\mathrm{D}$ \\
2 & 292 & 0.793 & 2.68 & $\mathrm{~F}$ \\
2 & 298 & 0.828 & 2.75 & $\mathrm{D}$ \\
\hline
\end{tabular}

\section{CONCLUSIONS}

Both axial and lateral chromatic aberrations affect the performance 3J GalnAs/GaAs/Ge concentrator solar cells. The extent of this effect depends on the size, material and quality of the Fresnel lens and its focal length. The work described herein is based on a specific Fresnel lens and is not representative of all lenses in general. Thick Fresnel lenses are desirable from the standpoint of rigidity but they suffer from optical absorption bands that occur in the region of the spectral response of the $\mathrm{Ge}$ bottom cell in the $3 \mathrm{~J}$ stack. FF changes, $\Delta F F$, due to focused and slightly defocused light can cause a variation in maximum power as high as $6.5 \%$. Which type of aberration has the most effect on the solar cell power output needs to be determined in more detail although lateral aberration can play a big role if a lens is distorted.

\section{REFERENCES}

[1] R.R. King et al. "Metamorphic III-V Materials, Sublattice Disorder, and Multijunction Solar Cell Approaches with Over 37\% Efficiency," to be Proc. 19th European Photovoltaic Solar Energy Conference, Paris, France, June 7-11, 2004.

[2] R. Sherif et al. "The Performance \& Robustness of GalnP/InGaAs/Ge Concentrator Solar Cells In High Concentration Terrestrial Modules,"to be Proc. 19th European Photovoltaic Solar Energy Conference, Paris, France, June 7-11, 2004.

[3] R. Sherif et al. "High Concentration Tests of a Dense PV Array Using GalnP/GaAs/Ge Triple Junction Cells in the High Flux Solar Furnace," to be publ. In $3^{\text {rd }}$ World Conference on Photovoltaic Energy Conversion, Osaka, Japan, May 11-18, 2003.

[4] H. Cotal, et al. "Outdoor Operation of GalnP/GaAs/Ge Triple Junction Solar Cells Up to 1000 Suns," to be publ. In $3^{\text {rd }}$ World Conference on Photovoltaic Energy Conversion, Osaka, Japan, May 11-18, 2003.

[5] H. Cotal et al. "High Concentration Testing and Performance of Multijunction Solar Cells," Proc. $28^{\text {th }}$ IEEE Photovoltaic Specialists Conf. (IEEE, New York, 2002), 1612.

[6] L.W. James "Effects of Concentrator Chromatic Aberration on Multijunction Cells," Proc. $1^{\text {st }}$ World Conference on Photovoltaic Energy Conversion, Waikoloa, Hawaii, December 5-9, 1994.

[7] M.J. O'Neil “Color-Mixing Lens for Solar Concentrator System and Methods of Manufacture and Opreation Thereof," U.S. Patent $6,031,179,2000$

[8] N. H. Karam et al. "Development and Characterization of High Efficiency GalnP/GaAs/Ge Dual- and Triple Junction Solar Cells," IEEE Trans ED (1999) $2116-2125$. 